\title{
Choroidal Ischemia Sparing the Watershed Zone following Intra-Arterial Chemotherapy for Retinoblastoma
}

\author{
David Arturo Ancona-Lezama ${ }^{a}$ Lauren A. Dalvin ${ }^{a}$ d J. Antonio Lucio-Alvarez ${ }^{a}$ \\ Pascal Jabbour ${ }^{b, c}$ Carol L. Shields ${ }^{a}$ \\ ${ }^{a}$ Ocular Oncology Service, Wills Eye Hospital, Thomas Jefferson University, Philadelphia, PA, USA; \\ ${ }^{b}$ Department of Neurovascular and Endovascular Surgery, Thomas Jefferson University, Philadelphia, PA, USA; \\ 'Department of Neurological Surgery, Thomas Jefferson University, Philadelphia, PA, USA; \\ ${ }^{\mathrm{d}}$ Department of Ophthalmology, Mayo Clinic, Rochester, MN, USA
}

\section{Established Facts}

- Intra-arterial chemotherapy (IAC) has an important role in the management of advanced cases of retinoblastoma and after failure of systemic chemotherapy.

- Choroidal ischemia is a known complication of IAC, occurring in 1-25\% of patients.

\section{Novel Insights}

- A unique form of choroidal ischemia following IAC, sparing the watershed zone and permitting visual acuity, is described.

\section{Keywords}

Choroidal ischemia · Intra-arterial chemotherapy · Retina •

Retinoblastoma - Watershed zone

\section{Abstract \\ Purpose: Intra-arterial chemotherapy (IAC) has become a mainstay in the management of retinoblastoma, especially in advanced or refractory disease. However, IAC is not with-}

out complications, and chemotherapy toxic effects can lead to partial or complete choroidal ischemia, often causing vision loss. Methods: This is a case report. Results: A 4-monthold girl with bilateral retinoblastoma was treated with secondary IAC (melphalan $5 \mathrm{mg}$ ) for recurrent tumor following intravenous chemotherapy. One month later, complete tumor control was achieved. However, she demonstrated broad choroidal ischemia in the nasal and temporal quadrants but sparing of the watershed zone superior and infe-

\section{KARGER}

(c) 2018 S. Karger AG, Basel

E-Mail karger@karger.com

www.karger.com/oop
Carol L. Shields, MD

Ocular Oncology Service, Wills Eye Hospital, Thomas Jefferson University 840 Walnut Street, Suite 1440

Philadelphia, PA 19107 (USA)

E-Mail carolshields@gmail.com 
rior to the optic disc and in the papillomacular region. Fluorescein angiography revealed poor perfusion of the choriocapillaris with visibility of the large choroidal vessels in the nasal and temporal areas but preserved perfusion of the watershed zone. The watershed zone remained intact on the 10-month follow-up, and the final visual acuity was fix and follow without strabismus. Conclusion: The pathophysiology of choroidal ischemia is not well understood, but the fortuitous watershed zone preservation in this case could represent uneven distribution of the chemotherapeutic drug, resulting in partial chemo-dilution of the medication in the watershed region, which represents the final downstream overlapping choroidal perfusion from both medial and lateral posterior ciliary arteries.

(c) 2018 S. Karger AG, Basel

\section{Introduction}

Intra-arterial chemotherapy (IAC) plays an essential role in the primary and secondary management of advanced retinoblastoma. Among its several uses, IAC has proven to be a powerful tool for globe salvage following failure of other therapies, particularly intravenous chemotherapy [1]. Despite the efficacy of IAC, serious local and systemic adverse events can occur [2]. Previously reported ophthalmic complications include eyelid/orbital edema, blepharoptosis, cutaneous erythema, strabismus, branch/central retinal artery occlusion, ophthalmic artery spasm or occlusion, choroidal ischemia, optic neuropathy, vitreous hemorrhage, and others [1, $3]$. In this case report, we focus specifically on choroidal ischemia.

The posterior ciliary arteries (PCAs) are responsible for the choroidal blood supply, particularly to the postequatorial region $[4,5]$. The ophthalmic artery spawns typically 2 (48\%) or 3 (39\%) main PCAs, and they further subdivide into the long PCAs $(n=2$, medial and lateral) and short PCAs $(n=10-20)$ [5]. The short PCAs then perforate the sclera in the circumpapillary region to provide choroidal perfusion in broad sectoral regions, up to the equator. There is overlap of choroidal perfusion between the main medial and lateral PCA routes, called the watershed zone [5]. This watershed zone varies but represents dual choroidal perfusion and typically is found between the fovea and nasal border of the optic disc as an irregular vertical strip, extending superior and inferior to the optic disc. This can be transiently visualized on fluorescein angiography (FA) during the early choroidal phase as a vertical hypofluorescent strip produced by patchy filling of the peripheral choriocapillaris lobules [5]. Due to the transient choroidal phase on FA, the watershed zone is only visible in $<45 \%$ of normal studies [4].

In an early analysis of FA outcomes following IAC for retinoblastoma with 55 ophthalmic artery catheterizations in 24 eyes, Bianciotto et al. [3] found angiographic evidence of retinal vascular abnormalities in 7 eyes (13\%) and choroidal vascular abnormalities in 6 eyes $(11 \%)$. The choroidal vascular abnormalities included reduced perfusion in a sectoral $(n=5)$ or diffuse $(n=1)$ pattern, but none with watershed zone sparing [3]. In a more recent analysis, Shields et al. [1] found that the combined incidence of ophthalmic, retinal, and choroidal ischemia has decreased to $1 \%$, possibly due to changes in the technique of IAC administration. Herein, we describe a unique post-IAC finding of choroidal ischemia with watershed zone sparing.

\section{Case Report}

A 4-month-old girl was referred for bilateral familial retinoblastoma. She had a family history of unilateral retinoblastoma in her father, treated with enucleation. She did not have a personal or family history of any coagulation disorders. On evaluation elsewhere, she demonstrated 2 small retinoblastomas in the right eye (OD), measuring 10 and $1.5 \mathrm{~mm}$ in basal diameter, and 1 retinoblastoma in the left eye (OS), measuring $13 \mathrm{~mm}$ in basal diameter. The patient was treated with 3 cycles of systemic chemotherapy consisting of vincristine $(0.05 \mathrm{mg} / \mathrm{kg})$, etoposide $(10 \mathrm{mg} / \mathrm{kg}$ divided in 2 days), and carboplatin $(18.6 \mathrm{mg} / \mathrm{kg})$. After the first cycle, she developed transitory vocal cord paresis that was attributed to vincristine, requiring a dose reduction for the second and third chemotherapy cycles. Following the third cycle of systemic chemotherapy, the patient was referred to our service for further management.

Examination under anesthesia confirmed bilateral partially treated retinoblastomas without retinal detachment, vitreous seed, or subretinal seed. The small tumors were consolidated with transpupillary thermotherapy (TTT) in combination with 3 additional monthly cycles of the same systemic chemotherapeutic agents previously used. At the 2-month follow-up, after the sixth cycle of systemic chemotherapy, there was recurrence of the macular retinoblastoma OS, and, additionally, there were 2 new tumors, with diameters of $1 \mathrm{~mm}$ and $400 \mu \mathrm{m}$, respectively (Fig. 1a, b). For management of these 3 active tumors, TTT versus IAC (melphalan $5 \mathrm{mg}$ ) was considered, but concern for immediate central vision loss from foveal TTT caused us to recommend IAC.

Following 1 cycle of IAC OS, reported to be uneventful by the treating neurosurgeon, tumor regression was achieved. The ophthalmic artery was reported patent before and right after the procedure with adequate delivery of the chemotherapy infusion. However, there was extensive retinal pigment epithelium atrophy nasally and temporally consistent with chemotherapy-induced choroidal ischemia (Fig. 1c). Optical coherence tomogra- 

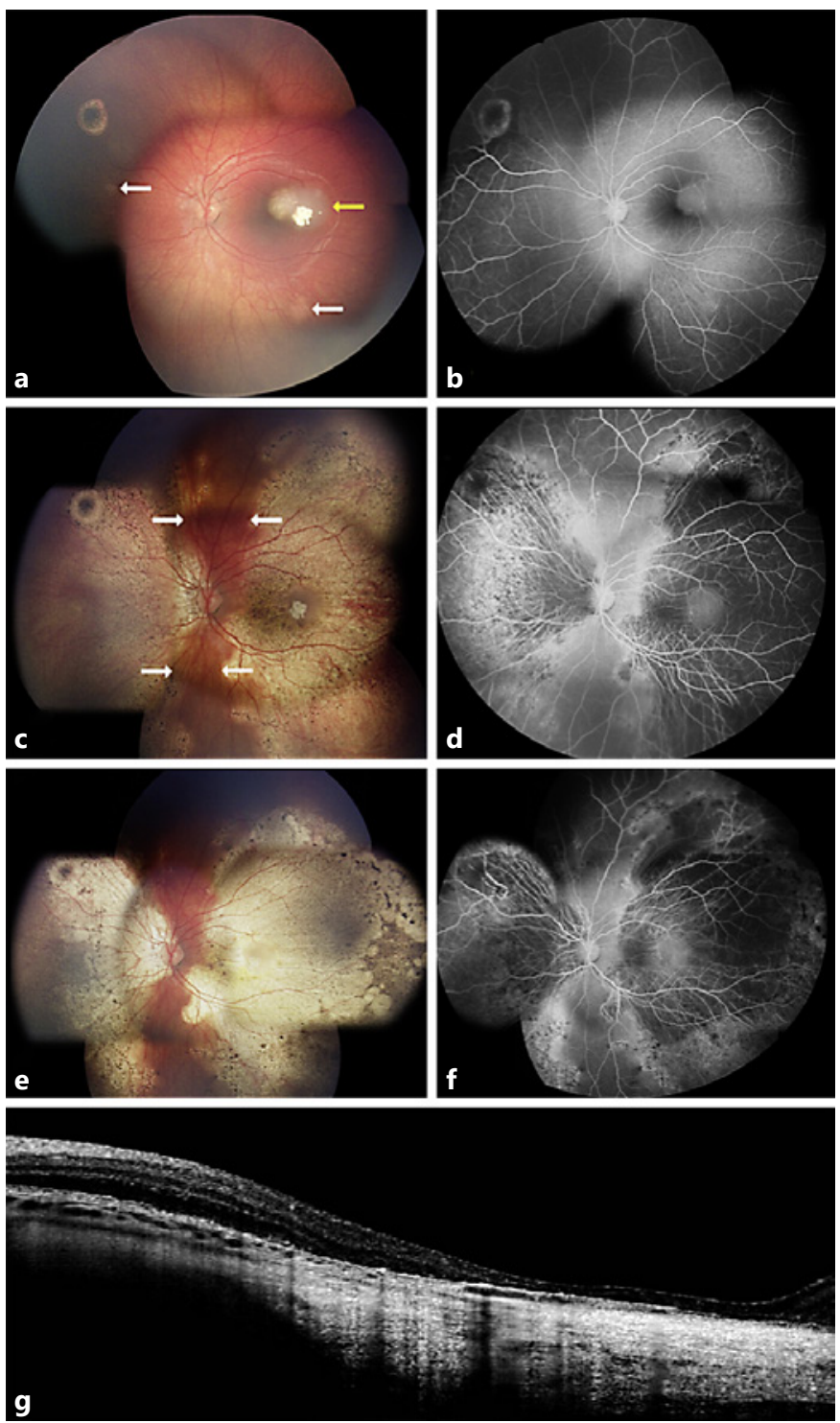

Fig. 1. a A 4-month-old girl demonstrated recurrent retinoblastoma in the temporal macula (yellow arrow) and 2 new tumors (white arrows) in the left eye after systemic chemotherapy and transpupillary thermotherapy consolidation. b Fluorescein angiography (FA) confirmed slight hyperfluorescence of the tumors and intact retinal and choroidal perfusion. The patient was treated with intra-arterial chemotherapy using melphalan $5 \mathrm{mg}$. c At 1-month follow-up, all tumors demonstrated regression. Broad choroidal ischemia with retinal pigment epithelial (RPE) atrophy was noted nasally and temporally but spared the watershed zone (white arrows) as a vertical strip through the papillomacular region. d On FA, hypofluorescent regressed tumors were noted, and the retinal perfusion was intact. However, there was broad choriocapillaris hypoperfusion nasally and temporally, with intact choriocapillaris in the watershed zone of the papillomacular region. e At 10 months, progression of choroidal atrophic changes and RPE alterations were observed with preservation of the watershed zone clinically, on FA (f), and by optical coherence tomography $(\mathbf{g})$. Retinal and choroidal atrophy are evident temporally, with preservation of retinal layers and choroid in the papillomacular bundle area. phy showed thin choroid, and FA revealed poor perfusion of the choriocapillaris with visibility of large choroidal vessels in the nasal and temporal ischemic areas. However, there was preservation of all choroidal vascularity in the watershed zone, as a vertical region superior and inferior to the optic disc and in the papillomacular region (Fig. 1d). Retinal perfusion appeared intact. Additional IAC was not administered due to the choroidal vascular toxicity. At 10 months post-IAC, tumor control persisted, but progressive choroidal and retinal pigment epithelium atrophy nasally and temporally, sparing the watershed zone with preservation of the papillomacular bundle, was noted on FA (Fig. 1e, f) and optical coherence tomography (Fig. 1g). Binocular vision allowed her to distinguish a grain of rice, and visual acuity was fix and follow OD and OS. The eyes were orthotropic even though the parents reported mild intermittent left esotropia for $5 \%$ of the day, and the child was able to recognize large toys with the affected eye. Occlusive patching for amblyopia prevention was instituted.

\section{Discussion}

A watershed zone, in medical terms, is an area supplied by the most distal portions of 2 nonanastomosing arterial circulations [6]. Watershed zones are found in the brain, heart, kidneys, intestines, and choroid $[5,7]$. The watershed zone can be both protective and vulnerable. For example, in occipital brain infarction, central macular vision can be spared due to the dual circulation (watershed zone) of the posterior and middle cerebral arteries. However, the watershed zone can be vulnerable to ischemia, especially during times of severe systemic hypotension, because the most distal arteriolar branches have the lowest perfusion pressure [8]. The main choroidal watershed zone lies between the areas supplied by the medial and temporal PCAs, typically found on either side of or encompassing the optic disc and as a vertical strip superiorly and inferiorly to the optic disc $[6,9]$. Here, we describe a unique case of choroidal ischemia secondary to IAC in which the watershed zone was spared.

Choroidal ischemia occurs in $1-25 \%$ of patients treated with IAC [1-3]. As opposed to retinal vascular abnormalities, which appear a median of 1 month after IAC, clinical changes from choroidal ischemia can progress slowly over time and are usually diagnosed several months after IAC is administered [3]. In our experience, choroidal atrophy can be diffuse, involving nearly the entire choroid, or can be sectoral, involving only a portion of the fundus. The pathophysiology of choroidal ischemia is currently unknown, with some hypothesizing that it could be due to catheter-related endothelial trauma with downstream microemboli, drug-related 
microemboli precipitation, or straightforward drug-related endothelial toxicity to the vascular tree [3].

Direct catheter trauma seems unlikely in this case because the catheter was advanced only to face the ostium of the ophthalmic artery. Catheter-related damage can occur if a catheter is advanced into the proximal ophthalmic artery, and this maneuver could decrease perfusion pressure to the entire vascular tree, including the choroid and retina, leading to hypoperfusion vulnerability of the watershed zone, which was not observed in this case. However, intra-arterial catheter thrombi formation secondary to endothelial damage could shed downstream microemboli, with only the overlapping watershed zone receiving adequate perfusion. Another theory on watershed zone sparing could be related to direct drug toxicity on the endothelium, affecting the most proximal choroidal lobules with the highest concentration, and, through downstream dilution, causing less damage to the peripheral overlapping watershed zone.

Studies in a nonhuman primate model revealed endothelial cell smudging, leukostasis, and terminal vascular occlusion following treatment with IAC using melphalan or carboplatin [9]. There is laboratory evidence that high doses of melphalan in vitro cause cell death in this manner, while low doses inhibit cell death [9]. If an area, such as the watershed zone, is exposed to only a very small amount of downstream melphalan, it could actually be more likely to survive compared to those areas upstream that receive the highest dose. We hypothesize that the drug(s) might not travel into the end arterioles in the watershed zone due to diffusion through or deposition in the more proximal vasculature. The theory of drug deposition is supported by the histopathologic discovery of birefringent material in the vascular lumens of eyes that have undergone enucleation following IAC [10]. Other mechanisms resulting in uneven drug distribution, such as laminar blood flow and branching vascular topology, as observed in the kidney, could also be at play [11].

Perfusion of the watershed zone in this case illustrates that despite the large areas of nasal and temporal choriocapillaris ischemia, there is continued perfusion of the large choroidal vessels, and there is clinical and angiographic evidence of overlapping watershed choriocapillaris perfusion. Admittedly, the pathophysiology of choroidal ischemia following IAC is not well understood, but the watershed zone sparing could be a clue that indicates 2 mechanisms, either uneven drug distribution into the PCAs, with sparing of the duplicate per- fusion, or direct chemotherapy-induced endothelial toxicity to the proximal branches with downstream dilution to the overlapping vascular bed. The preserved patency of the ophthalmic artery before and immediately following the IAC procedure along with the uneven fundus findings support a hypothesis that the watershed pattern of this complication is secondary to a medication-related side effect. Further studies are needed to find similar cases of watershed zone sparing. Potentially, alterations in technique, drug, or dosage could minimize this vascular event that resulted in broad choroidal ischemia. Such ischemia could cause large scotomas and decreased visual acuity, but preservation of the papillomacular bundle, as in this case, could allow for preservation of some useful vision.

\section{Statement of Ethics}

The subject provided consent to the publication of this case report and its associated images. No IRB approval was required for the publication of this case report.

\section{Disclosure Statement}

No authors have any conflicts of interest.

\section{Funding Sources}

Support provided in part by the Eye Tumor Research Foundation, Philadelphia, PA (C.L.S.), an unrestricted grant from Research to Prevent Blindness, Inc. (L.A.D.), the Heed Ophthalmic Foundation (L.A.D.), and a grant from the VitreoRetinal Surgery Foundation (L.A.D.). The funders had no role in the design and conduct of the study, in the collection, analysis and interpretation of the data, and in the preparation, review or approval of the manuscript. Carol L. Shields, MD, has had full access to all the data in the study and takes responsibility for the integrity of the data and the accuracy of the data analysis.

\section{References}

1 Shields CL, Manjandavida FP, Lally SE, Pieretti G, Arepalli SA, Caywood EH et al. Intraarterial chemotherapy for retinoblastoma in 70 eyes: outcomes based on the international classification of retinoblastoma. Ophthalmology. 2014 Jul;121(7):1453-60.

2 Shields CL, Bianciotto CG, Jabbour P, Griffin GC, Ramasubramanian A, Rosenwasser R et al. Intra-arterial chemotherapy for retinoblastoma: report No. 2, treatment complications. Arch Ophthalmol. 2011 Nov;129(11):1407-15. 
3 Bianciotto C, Shields CL, Iturralde JC, Sarici A, Jabbour P, Shields JA. Fluorescein angiographic findings after intra-arterial chemotherapy for retinoblastoma. Ophthalmology. 2012 Apr;119(4):843-9.

4 Giuffre G: Main posterior watershed zone of the choroid. Variations of its position in normal subjects. Doc Ophthalmol. 1989 Jun; 72(2):175-80.

5 Hayreh SS. Physiological anatomy of the choroidal vascular bed. Int Ophthalmol. 1983 Feb;6(2):85-93.
6 Hayreh SS: Posterior ciliary artery circulation in health and disease: the Weisenfeld lecture. Invest Ophthalmol Vis Sci. 2004 Mar;45(3): 749-57; 748.

7 Torvik A. The pathogenesis of watershed infarcts in the brain. Stroke. 1984 Mar-Apr; 15(2):221-3.

8 McFadzean R, Brosnahan D, Hadley D, Mutlukan E. Representation of the visual field in the occipital striate cortex. Br J Ophthalmol. 1994 Mar; 78(3):185-90.

9 Steinle JJ, Zhang Q, Thompson KE, Toutounchian J, Yates CR, Soderland C et al. Intraophthalmic artery chemotherapy triggers vascular toxicity through endothelial cell inflammation and leukostasis. Invest Ophthalmol Vis Sci. 2012 Apr;53(4):2439-45.
10 Eagle RC Jr, Shields CL, Bianciotto C, Jabbour P, Shields JA. Histopathologic observations after intra-arterial chemotherapy for retinoblastoma. Arch Ophthalmol. 2011 Nov; 129(11):1416-21.

11 Postnov DD, Salomonsson M, Sorensen CM, Sosnovtseva O. A simple method to ensure homogeneous drug distribution during intrarenal infusion. Am J Physiol Renal Physiol. 2017 Mar;312(3):F543-8. 